\title{
SMALL MOLECULES TARGETING THE VANILLOID RECEPTOR COMPLEX AS DRUGS FOR INFLAMMATORY PAIN
}

Rosa Planells-Cases ${ }^{1}$, Carolina García-Martínez ${ }^{1}$, Miriam Royo ${ }^{2}$, Enrique Pérez-Payá ${ }^{3}$, Cristina Carreño ${ }^{4}$, Fernando Albericio ${ }^{2}$, Angel Messeguer $^{3}$, and Antonio FerrerMontiel $^{1 *}$

${ }^{1}$ Instituto de Biología Molecular y Celular, Universidad Miguel Hernández, Av. Ferrocarril s/n, 03202 Elche, Alicante, Spain; ${ }^{2}$ Department of Organic Chemistry. Parc Cientific de Barcelona, Universidad de Barcelona, Baldiri Reixac 10-12, 08028 Barcelona, Spain. ${ }^{3}$ Dpt. of Biological Organic Chemistry, IIQAB-CSIC, C/Jordi Girona 11-18, 08034 Barcelona, Spain; ${ }^{4}$ DiverDrugs, SL, C/Santa Eulalia 242, 08902 L’Hospitalet de Llobregat, Barcelona, Spain. *Correspondence. 
Contents

1. Introduction

2. The TRP receptor family

3. The TRPV1 receptor

4. Pharmacology of TRPV1

5. TRPV 1 agonists

6. TRPV1 endogenous ligands

7. TRPV1 competitive antagonists

8. TRPV1 non-competitive antagonists

9. Hybrid TRPV1-cannabinoid modulators

10. The receptor complex as drug target

11. Perspective 


\section{Introduction}

Pain is an unpleasant sensation primarily designed to avoid tissue damage. Pain is considered a disease that needs aggressive physician treatment. Remarkably, the number of patients suffering pain has been increasing up to half a billion cases per year, having a tremendous impact on many quality-of-life measures (1). Undertreated or poorly treated pain is debilitating, and results in loss of work, productivity, family crisis, depression and/or suicide (2,3). Currently, it is estimated that at least $50 \%$ of individuals seeking treatment are unsatisfied with their present pain management options. Thus, the economic and medical costs of inadequate pain therapy in the community are vast.

Although pain has been traditionally considered as a unique and homogeneous pathology, cumulative progress has established that pain is an extremely complex and dynamic process involving multiple, interrelated neurotransmitter/neuromodulator systems in the peripheral nervous system. Indeed, as many as 15 neurotransmitters have been implicated in diverse aspects of pain-processing pathways (4-6). Two major types of pain are widely recognized, namely neuropathic and inflammatory. Nerve injury due to infection, autoimmune disease, trauma, and viral infections underlie the etiology of neuropathic pain. Damaged fibres ectopically discharge augmenting nerve activity, which induces hyperexcitability at the level of the spinal cord leading to burning-like pain.

At variance with neuropathic pain, inflammatory pain is produced in response to tissue damage $(4,7)$. Inflammatory pain involves various painful responses resulting from peripheral tissue injury and/or inflammation produced by trauma, infection, surgery, burns or diseases with an inflammatory component. In most cases, inflammatory pain is mediated by pro-algesic mediators such as protons, histamine, cytokines, prostaglandins, neurokinins, chemokines and ATP (4,7). These molecules 
sensitise neurons in the pain pathway (known as nociceptive neurons or nociceptors), either by directly modulating the sensitivity of membrane receptors or up-regulating intracellular signalling cascades. Nociceptors are sensitized by activation of both PKC and PKA pathways, which lead to phosphorylation of sensory receptors involved in nociceptive function $(8,9)$. As a result, strong sensory signalling is conveyed to the spinal cord and subsequently to specific brain regions leading to pain sensation. This phenomenon is known as peripheral sensitization. In addition, synaptic and metabolic changes at the level of the spinal cord may occur leading to the so-called central sensitization which further augments the pain sensation $(4,6)$. A major hallmark of inflammatory pain is thermal and mechanical hyperalgesia. Depending on the duration inflammatory pain is classified as acute or chronic.

The molecular components and mechanisms involved in inflammatory pain transduction are now starting to be unfolded. The identification and cloning of the TRPV1 channel represented a significant step in the clarification of the molecular mechanisms underlying transduction of noxious chemical and thermal stimuli by peripheral nociceptors. There is growing evidence that peripheral sensitization is largely mediated by excessive excitation of TRPV1 receptors $(10,11)$. Because of its central implication in hyperalgesia, the TRPV1 receptor has emerged as a key therapeutic target for inflammatory pain management.

\section{The TRP receptor family}

The Transient Receptor Potential (TRP) gene family is formed by more than 20 different gene products that result in non selective cation channels that play a wide diversity of physiological functions. TRP channels are divided into three subfamilies: TRPC, TRPV and TRPM that have a common topology of six transmembrane segments 
(S1-S6) with a pore region between the fifth and sixth segment, and cytoplasmic $\mathrm{N}$ - and C-termini. TRPV and TRPC contain two to four ankyrin domains that are thought to interact with the cytoskeleton (12). The first cloned TRP, a TRPC channel from Drosophila resulted essential for visual transduction. In the worm C. elegans, TRPC2 is required for transduction of pheromone-response of vomeronasal neurons [13]. These are metabotropic receptors that act through G-protein coupled receptors via the phospholipase $\mathrm{C}$ pathway releasing $\mathrm{Ca}^{2+}$ from the endoplasmic reticulum trough inositol receptors (14). In contrast, the TRPV family are ionotropic receptors activated by noxious stimuli, for instance, TRPV1 gates in response to noxious temperature $\left(\geq 42^{\circ} \mathrm{C}\right.$, vanilloids, acidic $\mathrm{pH}$, and pro-algesic substances (15). Three additional TRPV channels have been cloned that activate at different temperature thresholds: TRPV 3 from $33^{\circ} \mathrm{C}$ (16), TRPV2 to $55^{\circ} \mathrm{C}$, and TRPV4 to warm temperatures and to osmolarity. Recently, two receptors of the TRPM family that respond to cold temperatures have been cloned. TRPM8 and ANKTM1 activate at $25^{\circ} \mathrm{C}$ and $17^{\circ} \mathrm{C}$, respectively. Therefore, TRP channels constitute a family of sensory receptors.

\section{The TRPV1 receptor}

It has been long known that a subset of peripheral sensory neurons are excited by vanilloids. Cumulative studies indicate that vanilloid sensitive nociceptors are quite heterogeneous morphological, neurochemical and functionally. In general they are peptidergic, small diameter neurons that give rise to unmyelinated $\mathrm{C}$ fibers, with best correlation with Substance P, although some Ad fibers have also been found sensitive to vanilloid derivatives (17). TRPV1 expression has been found in dorsal root and trigeminal ganglia, in dorsal horn of spinal cord and caudal nucleus of spinal trigeminal complex by in situ hybridization, immunocytochemical analysis, and drug binding 
assays $(15,18)$. In dorsal root ganglia, more than $50 \%$ of neurons are TRPV1 positive. Immuno-specific staining of TRPV1 has been observed in the plasma membrane, Golgi and small clear vesicles (19). This ubiquitous intracellular distribution may be relevant for inflammation-induced sensitization.

TRPV1 is also present in various brain nuclei, as well as in non-neuronal tissues, as exemplified by urinary bladder epithelium, smooth muscle and epidermal keratinocytes (20-22). TRPV1 is expressed in most of the afferent nerve fibres in the rodent gastrointestinal tract and some of the vagal afferents, however is still unknown whether it plays a role in gut sensation. TRPV1 is found not only in afferent fibres within the urinary bladder but also in submucosa, mucosa and epithelial cells lining the bladder $(21,23)$. Thus, these observations provide a mechanism for the variety of functions exerted by vanilloids in non-neuronal tissues. For example, vanilloidmediated TRPV1 refractoriness is effective in visceral hypersensitivity. Resiniferotoxin and the capsaicin-analogue SDZ249-665 effectively attenuate inflammatory bladder hyperalgesia and visceral pain responses to intraperitoneal acetic acid in rats. Taken together, these findings substantiate the notion that TRPV1 plays a role in neurogenic inflammation and visceral pain.

An expression-cloning strategy, using capsaicin as an specific agonist, unveiled the molecular identity of TRPV1. Heterologous expression of TRPV1 cDNA results in capsaicin-activated inward currents that recapitulate most of the functional properties of those described for the capsaicin and heat-activated receptors of dorsal root ganglion neurons. Receptor functional analysis demonstrated that TRPV1 is a non-selective cation channel activated by vanilloids, that exhibits a time- and $\mathrm{Ca}^{2+}$-dependent outward rectification followed by a long-lasting refractory state (during which the cell does not respond to capsaicin and other stimuli). TRPV1 is also gated at temperatures higher 
than $42^{\circ} \mathrm{C}$. In addition, mild extracellular acidic $\mathrm{pH}$ potentiates vanilloid receptor activation by noxious heat and vanilloids, while strong acidic conditions $(\mathrm{pH}<6)$ directly activates the channel. A widely held tenet is that vanilloids and acidic $\mathrm{pH}$ decrease the threshold for heat activation from noxious to non-noxious temperatures. Therefore, TRPV1 integrates painful physical and chemical noxious stimuli.

Mice deficient in TRPV1 have been generated to validate TRPV1 as a therapeutic target. Physiological and behavioural studies of the knock out animals indicated a clear role of TRPV1 in thermal hyperalgesia, but not in mechanical hypersensitivity $(24,25)$. Intriguingly, a modest contribution of TRPV1 to thermal nociception was observed, perhaps due to developmental over-expression of other TRPV members that contribute to the final phenotype. These studies also showed that TRPV1 gene ablation does not modify neuropathic pain responses. Similar conclusions were reached by using pharmacological tools. In vivo inhibition of TRPV1 activity by non-competitive antagonists attenuated thermal nociception and hyperalgesia, and reduced neurogenic inflammation (26). Taken together, all these findings illustrate that TRPV1 is a widely expressed neuronal receptor that behaves as a gateway for pain transduction and, in turn, validate this receptor as a key therapeutic target for pain management.

\section{Pharmacology of the TRPV1 receptors}

Because of its involvement in the ethiology of inflammatory pain, pharmacological modulation of TRPV1 is a valuable strategy to mitigate pain sensation. Structure-function studies have revealed that the TRPV1 receptor is a molecular entity with various potential drug binding sites. These properties have prompted the discovery of different kinds of receptor modulators with the hope that they will be of clinical use 
to treat TRPV1 receptor dysfunction, especially in inflammatory conditions. However, because of the relevant role of this thermosensor in the physiology of nociceptors, complete abrogation of channel activity may lead to unwanted side-effects such as general thermal insensitivity. We next will summarize the efforts undertaken to develop three classes of TRPV1 modulators, namely agonists, competitive antagonists and channel blockers.

\section{TRPV1 agonists}

Agonists are molecules that gate the channel. Prolonged exposure of the receptor to the agonist, however, induces its closure by taking the protein to a desensitized state. Agonist-induced desensitization is dependent of the presence of extracellular $\mathrm{Ca}^{2+}$, as it is the process known as tachyphilaxia which is characterized by a profound run-down of TRPV1 responses upon repetitive agonist stimulation. Both desensitization and tachyphlaxia are of therapeutic value because of receptor down regulation. Paradoxically, agonist-induced neuronal death due to sustained receptor activation, especially under ischaemic conditions, is also useful for treating specific pain conditions.

The best known TRPV1 agonist family is that of vanilloids. Capsaicin is the typical structure of one class of vanilloid compounds, the capsacinoids (Fig....). There are another three known chemical classes of naturally occurring vanilloids: resiniferanoids, unsaturated dialdehydes and triprenyl phenols, whose typical representative structures are resiniferatoxin (RTX), isovelleral and scutigeral respectively (27).

Capsaicin is known to have three functional regions, an aromatic A region, where is optimal a parent homovanillyl (3-methoxy 4-hydroxybenzyl) group, a B 
region, known as the ester o amide linker, and the aliphatic $\mathrm{C}$ region, where a lipophilic octanyl or $p$-chlorophenetyl moiety is associated with the highest potency. The homovanillyl motif and amide bond regions contain dipolar groups, which are implicated in hydrogen bonding interactions. Analysis of the structure-activity relationship of numerous capsaicin analogues suggest that these polar regions are essential for maintaining pungency, which could be alternatively expressed as maintaining the excitation of sensory neurons. In contrast, the aliphatic chain in the $\mathrm{C}$ region, which has an optimal chain length of 8-10 carbon atoms, is presumed to interact hydrophobically with its receptors (27). Although a receptor model has been suggested based on the structure-activity relationships of the capsaicin analogs, structural elements in TRPV1 that confer specific interactions with vanilloids have not been characterized. Moreover, unlike other ligand-gated channels that produce fast synaptic transmission, vanilloids are known to act on the TRPV1 receptor from the intracellular site (28). Recently, a region spanning the third transmembrane domain (S3) in TRPV1 was found to be essential for ligand binding, presumably by hydrophobic interactions with capsaicin (29). Complementary regions in TRPV1, predominantly those located in both cytosolic tails, have also been implicated in structuring the agonist binding site (28).

Vanilloid compounds have been considered as potential analgesics and have been extensively used in medicine. Capsacinoids are traditionally indicated for relieving toothache. Capsaicin is a standard ingredient in a variety of over-the-counter drugs used worldwide to relieve muscle ache, as an example of the therapeutic value of the excitation that it provokes. Desensitization to capsaicin has a clear therapeutic potential ameliorating urinary bladder overactivity, uraemic itching associated with renal failure, urinary incontinence and inflammatory bowel disease. Additionally, capsaicin-induced neurotoxicity has already been shown to be useful in the treatment of neuropathic pain 
such as herpes-zoster related neuropathic pain, diabetic neuropathy, and postmastectomy pain (27).

A major shortcoming of therapeutic capsaicin is burning sensation and irritation that it produces. Structure-activity relationship studies of vanilloids have led to the development of more potent, orally active, vanilloid-like molecules such as SDZ249482 and KR-25018. However, these compounds still exhibit irritant side effects. Thus, a drug with long lasting desensitisation and no pungency promises to be of great clinical value. There are naturally and synthetic non pungent capsaicin analogs, like capsiate, olvanil, glycerol nonivamide, and scutigeral, but their low efficacies prevent their clinical development. However, ongoing clinical trials with resiniferatoxin (RTX) (Fig. 1), a potent capsaicin analogue isolated from Euphorbia resinifera, indicate that such vanilloids may in fact be useful (27).

The specific binding of RTX to the capsaicin binding site in dorsal root ganglia has been demonstrated with labeled $[$ H $\mathrm{H}]-\mathrm{RTX}$. The pharmacophoric groups of RTX have not yet been clearly defined, although structure-activity studies suggest that the $\mathrm{C}_{20}$-homovanillic moiety, the $\mathrm{C}_{3}$-keto group, and the orthoester phenyl group on ring $\mathrm{C}$ are crucial structural elements responsible for the extremely high potency of RTX. RTX is being developed as an ultrapotent sensory neuron desensitising agent for the treatment of urinary urge incontinence and the pain associated with diabetic neuropathy and appears to be superior to capsaicin in terms of its tolerability profile (27). The unquestionable therapeutic potential of RTX is seriously hampered by its limited availability from natural sources and the complexity of its chemical synthesis, as evidenced by the more than 44 reaction steps required for its total synthesis. Nonetheless, clinical trials with RTX are currently ongoing. 


\section{TRPV1 endogenous ligands}

Because TRPV1 is chemically gated the question of whether there are endogenous substances that gate the channel has emerged. There is cumulative evidence that endogenous vanilloid receptor agonists ('endovanilloids') exist and play a central role in the development inflammatory hyperalgesia. There is no doubt that these agents modulate the sensitivity of vanilloid receptors to thermal stimuli.

The first endogenous compound thought to be an endovanilloid was anandamide, a polyunsaturated fatty acid ( $\mathrm{N}$-arachidonoylethanolamine), activating both native and recombinant rat TRPV1 receptors (Fig. 2) (30). However, anandamide is also considered an endocannabinoid since activates both cannabinoid CB1 and CB2 receptors at lower concentrations than those needed to activate TRPV1.

Capsaicin-like substances are structurally similar to some eicosanoids, particularly those derived from the action of 5-lipoxygenase and 12-lipoxygenase and, indeed, arachidonic acid has been found capable of activating the rat TRPV1, and being most potent 12-hydroperoxy-eicosatetraenoic acid (12-HPETE) and leukotriene B4 (LTB4) (Fig. 2) (31). The binding site on TRPV1 is believed to occur, only by slow, passive diffusion. Eicosanoids are believed to function as 'intracellular endovanilloids', acting at TRPV1 receptors in the cells where they are synthesized.

Recent studies have led to the identification of another compound, characterized as $\mathrm{N}$-arachidonoyl-dopamine (NADA) proposed to act as a 'brain endovanilloid since relatively abundant in some brain areas where TRPV1 is also present (Fig. 2) (32). $\mathrm{NADA}$ is an endogenous anandamide analogue several times more potent than anandamide on TRPV1, yet still capable of activating CB1 receptors. Thus, the identification and characterization of endovanilloids may provide important clues to the development of innovative analgesic and anti-inflammatory drugs. 


\section{TRPV1 antagonists}

Competitive and non-competitive vanilloid antagonists are the two major classes of TRPV1 modulators being explored for therapeutic exploitation. The first class specifically seeks for compounds that bind to the agonist binding site and lock the channel in the closed, non-conductive state. Non-competitive antagonists, however, interact with additional drug binding sites located on the receptor structure. Of special interest are those acting as open channel blockers because they specifically recognize the overactivated species, i.e. pathological receptors.

\section{TRPV1 competitive antagonists}

The first competitive antagonist known, capsazepine was prepared by introducing a saturated seven-membered rigid ring system, which maintained a virtually orthogonal conformation between each $\mathrm{N}$-substituent in the thiourea structure (Fig. 3). Tetrahydrobenzazepine and tetrahydroisoquinoline thiourea derivatives have been prepared as antagonists by the replacement of the $p$-chlorophenethyl group with 3acyloxy-2-benzylpropyl groups. All of them are competitive vanilloid antagonists with a potency ranging from 0.2 to $4 \mu \mathrm{M}$. When administered in animal models of inflammatory pain these compounds exhibit analgesic activity, although their therapeutic index is still low (33). In addition, they exhibit limited blockade activity on heat-activated channels present in the neuronal surface.

Recently, a derivative of RTX, 5-Iodo-RTX, was shown to be a highly potent antagonist $\left(\mathrm{IC}_{50}, 3.9 \mathrm{nM}\right)$ of TRPV1 (Fig. 3). This compound exhibits analgesic activity in vivo and is currently in pre-clinical studies. As for RTX, a major drawback is the complexity and cost of its chemical synthesis from RTX, and its limited oral activity (34). 
Taking together, the hunt for better competitive vanilloid antagonists is a major goal of pharmaceutical companies. We should witness in the near future the blossom of these efforts reflected as increasing pipelines of lead compounds for analgesic drug discovery.

\section{TRPV1 non-competitive antagonists}

The first non-competitive antagonist introduced as a "functional capsaicin antagonist" was ruthenium red, which is an inorganic polyamine acting as a noncompetitive antagonist that binds to the pore region of the channel (Fig. 4) (27). The therapeutic use of ruthenium red is highly precluded because of its unspecific blockade activity.

Arginine rich hexapeptides such as RRRRWW- $\mathrm{NH}_{2}$ block recombinant TRPV1 channels expressed in Xenopus oocytes with submicromolar efficacy by a noncompetitive way (Fig. 4) (35). It was recently reported that they may also act through a competitive mechanism (36). Apart from the specific mechanism, their development as analgesic drugs is prevented by their pharmacological unsafety.

A novel class of non-competitive antagonists of TRPV1 was identified from the screening of a peptidomimetic-based library (26) (Fig. 4). Peptidomimetic molecules such as $\mathrm{N}$-alkylated glycines (also known as peptoids) constitute a family of non- natural compounds that exhibit interesting biological properties (37). Formally, peptoids result from the shift of the substituent present at $\alpha$-carbon atom in amino acids to the adjacent nitrogen atom. Nevertheless, although being isomers of peptides, peptoids show different structural features, such as the higher degree of conformational freedom and the absence of $\mathrm{CO}-\mathrm{NH}$ hydrogen bonds. These properties modify the steric 
interactions leading to the secondary structure present in peptides. Moreover, contrary to what occurs in peptides, peptoid backbones are achiral and protease resistant.

With regard to TRPV1, the screening of a library of trimers of $\mathrm{N}$-alkylglycines resulted in the identification of two molecules referred to as DD161515 \{N-[2-(2(Nmethylpyrrolidinyl)ethyl]glycyl]-[N-[2,4-dichlorophenethyl]glycyl]-N-(2,4dichlorophenethyl)glycinamide $\} \quad$ and $\quad$ DD191515 $\quad\{[\mathrm{N}-[3-$ N,Ndiethylamino)propyl]glycyl]-[N-[2,4-dichlorophenethyl]glycyl]-N-(2,4dichlorophenethyl)glycinamide\} that selectively block TRPV1 channel activity with micromolar efficacy, rivaling that characteristic of vanilloid-related inhibitors (Fig. 4). These compounds appear to be non-competitive TRPV1 antagonists that recognize a receptor site distinct from that of capsaicin. Intraperitoneal administration of both trialkylglycines into mice significantly attenuated thermal nociception as measured in the hot plate test. It is noteworthy that these compounds eliminated pain and neurogenic inflammation evoked by intradermal injection of capsaicin into the animal hindpaw, as well as the thermal hyperalgesia induced by tissue irritation with nitrogen mustard. In contrast, responses to mechanical stimuli were not modified by either compound. Modulation of sensory nerve fibers excitability appears to underlie the peptoid analgesic activity. Collectively, it seems that these trialkylglycine-based, non-competitive TRPV1 antagonists may likely be developed into analgesics to treat inflammatory pain. Further progress in this exciting field is being eagerly pursued and new and more potent and selective hits for drug development are being identified and characterized. For example, hydantoin-based compounds have been identified as potent blockers of TRPV1 channels. Notably, lead compounds that produce analgesia without affecting normal nociceptive responses have been developed. We await for the clinical validation of this sort of TRPV1 antagonists. 


\section{Hybrid cannabinoid-TRPV1 modulators}

Since TRPV1 is expressed in several brain regions, and next to CB1 receptors, it could be possible that some of the neurobehavioral effects of those endogenous compounds derive from the combined activation over at least, both receptors. The activation of these receptors often leads to similar pharmacological actions (e.g., antinociception, hypothermia, vasodilation, hypokinesia, and inhibition of cancer cell growth Although via different mechanisms, compounds capable of simultaneously activating two or more of these targets may have great therapeutic potential. Those are the "hybrid" agonists like 'arvanil' (N-[3-methoxy-4-hydroxy-benzyl]-arachidonamide). It presents an affinity for $\mathrm{CB} 1$ receptors comparable to that of AEA and also activates TRPV1 receptors more potently than anandamide and capsaicin (Fig. 5) (38). Thus, arvanil is much more potent than either anandamide or capsaicin (i) as an antiproliferative agent for human breast cancer cells, in a fashion sensitive to both CB1 and TRPV1 receptor antagonists, (ii) as a cannabimimetic agent in the mouse model 'tetrad', (iii) as a spinal analgesic and (iv) as a relaxant of mouse vas deferens. However, arvanil efficacy is not well balanced between TRPV1 and CB1 receptors and hence, new "hybrid" CB1/TRPV1 agonists with possible therapeutic importance should be developed. What is of great interest is to realize that TRPV1 and cannabinoid CB1 receptors have overlapping ligand recognition properties which may also have far reaching implications for vanilloid therapy.

\section{Modulators of the TRPV1 receptor signalling complex.}

An emerging concept is that ion channels are not isolated devices in the cell membrane, they are rather components of multiprotein complexes that play a key role in 
physiology and pathology. These macromolecular assemblies are high order signalling networks that balance external and internal signals giving rise to a response. Dysfunction of protein networks thus has deleterious consequences. These complexes are composed by a plethora of proteins that include receptor, adaptor, signalling, cytoskeletal and novel proteins. Assigned functions to these synaptic signalling assemblies are the biogenesis, distribution and clustering of receptors and their colocalization with signalling proteins for an efficient transduction of external cues. Thus the synaptic protein complex contains a variety of potential drug targets. The molecular identity and organization of the TRPV1 signalling complex is still elusive. Fastadvancing progress in this arena is uncovering the molecular identity of these elements which, in due turn, will be validated as therapeutic targets for drug intervention. Because most of these targets are protein complexes, the real challenge ahead would the discovery and development of small molecules that modulate protein-protein interactions, a yet unmet goal of current drug discovery.

\section{Perspective}

The unquestionable involvement of the TRPV1 receptor in pain transduction has thrust an interest on the development of antagonists of this neuronal receptor for pain management. This is apparently an easy task for current drug discovery platforms, although the real challenge is to develop antagonists that preserve the physiological activity of TRPV1 receptors while correcting over-active receptors. This is a condition to ensure normal pro-prioceptive and nociceptive responses that represent a safety mechanism to prevent tissue injury. Therefore, physiological constraints have to be included in the development and optimization of TRPV1 receptor antagonists to ensure their clinical utility as pain-killers. 
Acknowledgments. The authors thank all colleagues and members of their research and collaboration groups for their fundamental contribution to the results herein presented. Financial support from grants from MCYT, Fundació La Caixa, Fundació Marató TV3, and FIS are acknowledged. 


\section{References}

1. Russo, C.M. and Brose, W.G. Chronic Pain. Annu Rev Med, 1998, 49: 123-33.

2. Wolf, C. and Salter, M.W. Neuronal plasticity: increasing the gain in pain. Science 2000, 288: 1765-68.

3. Brower, V. New paths to pain relief. Nat Biotech, 2000, 18: 387-91.

4. Williams, M., Kowaluk, E.A. and Arneric, S.P. Emerging molecular approaches to pain therapy. J Med Chem, 1999, 42: 1481-500.

5. McCleskey, E.W. and Gold, M.S. Ion channels of nociception. Annu Rev Physiol, 1999, 61: 835-56.

6. Cesare, P. and McNaughton, P. Peripheral pain mechanisms. Curr Opin Neurobiol, 1997, 7: 493-99.

7. Wood, J.N. and Docherthy, R. Chemical activators of sensory neurons. Rev Physiol, 1997,$59 ; 457-482$.

8. Malmberg, A.B., Chen, C., Susumu, T. and Basbaum, A.I. Preserved acute pain and reduced neuropathic pain in mice lacking PKC?. Science, 1997, 278: 279-83.

9. Cui, M. and Nicoll, G.D. Cyclic AMP mediates the prostaglandin $E_{2}$-induced potentiation of bradykinin excitation in rat sensory neurons. Neuroscience, 1995, 66: 459-66.

10. Caterina, M.J. and Julius, D. The vanilloid receptor: a molecular gateway to the pain pathway. Annu Rev Neurosci, 2001, 24: 487-517.

11. Willis, W.D. Role of neurotransmitters in sensitization of pain responses. Ann N Y Acad Sci, 2001, 933; 142-56.

12. Montell, C., Birnbaumer, L. and Flockerzi, V. The TRP Channels, a remarkably functional family. Cell, 2002, 108: 595-98. 
13. Stowers, L., Holy, T.E., Meister, M., Dulac, C. and Koentges, G. Loss of sex discrimination and male-male aggression in mice deficient for TRP2 Science, 2002, 295: 1493-500.

14. Minke, B. and Cook, B. TRP channel proteins and signal transduction. Physiol Rev, 2002, 82: 429-72.

15. Caterina, M.J., Schumacher, M.A., Tominaga, M., Rosen, T,A., Levine, J.D. and Julius, D. The capsaicin receptor: a heat-activated channel in the pain pathway. Nature 1997, 389: 816-24.

16. Peier, M.A., Reeve, A.J., Andersson, D.A., Moqrich, A., Earley, T.J., Hergarden A.C., Story, G.M., Colley, S., Hogenesh, J.B., McIntyre, P., Bevan, S. and Patapoutian, A. Heat-Sensitive TRP Channel expressed in keratinocytes Science 2002, 296: 20462049.

17. Holzer, P. Capsaicin: cellular targets mechanisms of action, and selectivity for thin sensory neurons. Pharmacol Rev, 1991, 43: 143-01.

18. Tominaga, M., Caterina, M.J., Malmberg, A.B., Rosen, T.A., Gilbert, H., Skinner, K., Raumann, B.E., Basbaum, A.I. and Julius, D. The cloned capsaicin receptor integrates multiple pain-producing stimuli. Neuron, 1998, 21:531-43.

19. Guo, A., Vulchanova, L., Wang, J., Li, X. and Elde, R. Immunocytochemical localization of the vanilloid receptor (VR1): Relationship to neuropeptides, the P2X3 purinoceptor and IB4 binding sites. Eur J Neurosci, 1999, 11: 946-58.

20. Mezey, È., Tóth, Z.E., Cortright, D.N., Arzubi, M.K., Krause, J.E., Elde, R., Guo, A., Blumberg, P.M. and Szallasi, A. Distribution of mRNA for vanilloid receptor subtype 1 (VR1), and VR1-like immunoreactivity, in the central nervous system of the rat and human. Proc Natl Acad Sci USA, 2000, 97:3655-60. 
21. Birder, L., Kanai, A.J., de Groat, W.C., Kiss, S., Nealen, M.L., Burke, N.E., Dineley, K.E., Watkins, S., Reynolds, I.J. and Caterina, M.J. Vanilloid receptor expression suggests a sensory role for urinary bladder epithelial cells. Proc Natl Acad Sci USA, 2001, 98:13396-401.

22. Inoue, K., Koizumi, S., Fuziwara, S., Denda, S., Inoue, K. and Denda, M. Functional vanilloid receptors in cultured normal human epidermal keratinocytes. Biochem Biophys Res Commun, 2002, 291: 124-29.

23. Avelino, A., Cruz, C., Nagy, I. and Cruz, F. Vanilloid receptor 1 expression in the rat urinary tract. Neuroscience, 2002, 109: 787-98.

24. Caterina, M.J., Leffler, A., Malmberg, A.B., Martin, W.J., Trafton, J., PetersenZeitz, K.R., Koltzenburg, M., Basbaum, A.I. and Julius, D. Impaired nociception and pain sensation in mice lacking the capsaicin receptor. Science, 2000, 288; 306-13.

25. Davis, J.B., Gray, J., Gunthorpe, M.J., Hatcher, J.P., Davey, P.T., Overend, P., Harries, M.H., Latcham, J., Clapham, C., Atkinson, K. et al. Vanilloid receptor-1 is essential for inflammatory thermal hyperalgesia. Nature, 2000, 405: 183-87.

26. García-Martínez, C., Humet, M., Planells-Cases, R., Gomis, A., Caprini, M., Viana, F., De la Peña, E., Sanchez-Baeza, F., Carbonell, T., DeFelipe, C., Pérez-Payá, E., Belmonte, C. and Ferrer-Montiel, A.V. Attenuation of thermal nociception and hyperalgesia by VR1 blockers. Proc Natl Acad Sci USA, 2002, 99: 2374-79.

27. Szallasi, A. and Blumberg, P.M. Vanilloid (capsaicin) receptors and mechanisms. Pharmacol Rev, 1999, 51: 159-11.

28. Jung, J., Hwang, S.W., Kwak, J., Lee, S.Y., Kang, J.C., Kim, W.B., Kim, D. and Oh, U. Capsaicin binds to the intracellular domain of the capsaicin activated ion channel. J Neurosci, 1999, 19:529-538. 
29. Jordt, S.E. and Julius, D. Molecular basis for species-specific sensitivity to "hot" chili peppers. Cell, 2002, 108:421-30.

30. Zymunt, P.M., Peterson, J., Anderson, D.A., Chuang, H., Sorgard, M., Di Marzo, V., Julius, D. and Hogestatt, E.D. Vanilloid receptors on sensory nerves mediates the vasodilator action of anandamide. Nature, 1999, 400: 452-57.

31. Hwang, S.W., Kwak, J., Wang, M.H., Hwang, S.W., Kim, T.Y., Lee, L.Y. and Oh, U. Direct activation of capsaicin receptors by products of lipoxygenases: endogenous capsaicin-like substances. Proc Natl Acad Sci USA, 2000, 97: 6155-60.

32. Huang, S.M., Bisogno, T., Trevisani, M., Al-Hayani, A., De Petrocellis, L., Fezza, F., Tognetto, M., Petros, T.J., Krey, J.F., Chu, C.J. et al. An endogenous capsaicin-like substance with high potency at recombinant and native vanilloid VR1 receptors. Proc Natl Acad Sci USA, 2002, 99: 8400-05.

33. Park, H., Park M., Choi, J., Choi S., Lee, J., Park, B., Kim G., Suh, Y., Cho, H., Oh, U., Lee, J., Kim, H., Park, Y., Koh, H., Limc, K., Mohc, J. and Jewa, S. Synthesis of $\mathrm{N}, \mathrm{N}^{\prime}, \mathrm{N}^{\prime \prime}$-Trisubstituted Thiourea Derivatives and Their Antagonist Effect on the Vanilloid Receptor. Bioorg Med Chem Letters, 2003, 13: 601-04

34. Wahl, P., Foged, C., Tullin, S. and Thomsen, C. Iodo-Resiniferatoxin, a New Potent Vanilloid Receptor Antagonist. Mol Pharmacol, 2001, 59:9-15.

35. Planells-Cases, R., Aracil, A., Merino, J., Gallar, J., Pérez-Payá, E., Belmonte, C. and Ferrer-Montiel, A.V. Arginine rich peptides are blockers of VR-1 channels with analgesic activity. FEBS Lett, 2000, 481:131-36.

36. Himmel, H.M., Kiss, T., Borvendeg, S.J., Gillen, C. and Ille, P. The arginine-rich hexapeptide R4W2 is a stereoselective antagonist at the vanilloid receptor 1: $\mathrm{Ca}^{2+}$ imaging study in adult rat dorsal root ganglion neurons. J Pharmacol Exp Ther 2002, 301: 981-986. 
37. Horwell, D. C.et al. Use of a dipeptide chemical library in the development of nonpeptide tachykinin NK3 receptor selective antagonists. J Med Chem, 1996, 39, 16641675.

38. Di Marzo, V., Bisogno, T., De Petrocellis, L., Brandi, I., Jefferson, R.G., Winckler, R.L., Davis, J.B., Dasse, O., Mahadevan, A., Razdan, R.K., Martin, B.R. Highly selective $\mathrm{CB}(1)$ cannabinoid receptor ligands and novel $\mathrm{CB}(1) / \mathrm{VR}(1)$ vanilloid receptor “hybrid” ligands. Biochem. Biophys. Res. Commun., 2001, 281: 444-51. 


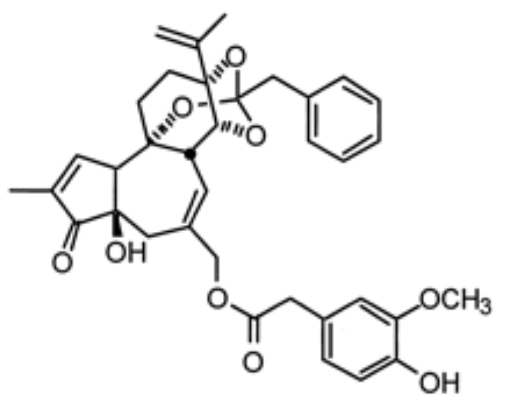

Resiniferatoxin

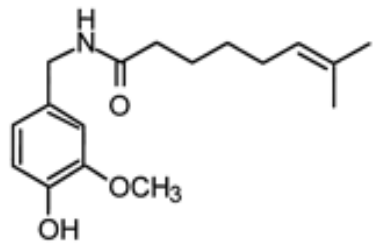

Capsaicin

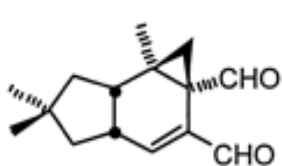

Isovelleral

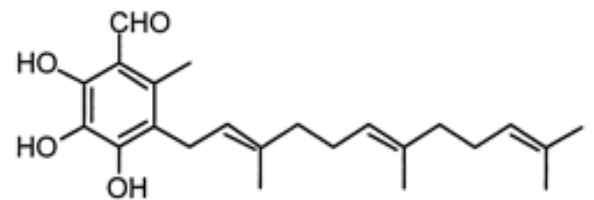

Scutigeral

Fig. Typical vanilloid structures: RTX, capsaicin, isovelleral, and scutigeral, representing the four known chemical classes of naturally occurring vanilloids, resiniferanoids, capsaicinoids, unsaturated dialdehydes, and triprenyl phenols, respectively. (Szallasi et al 1999) 


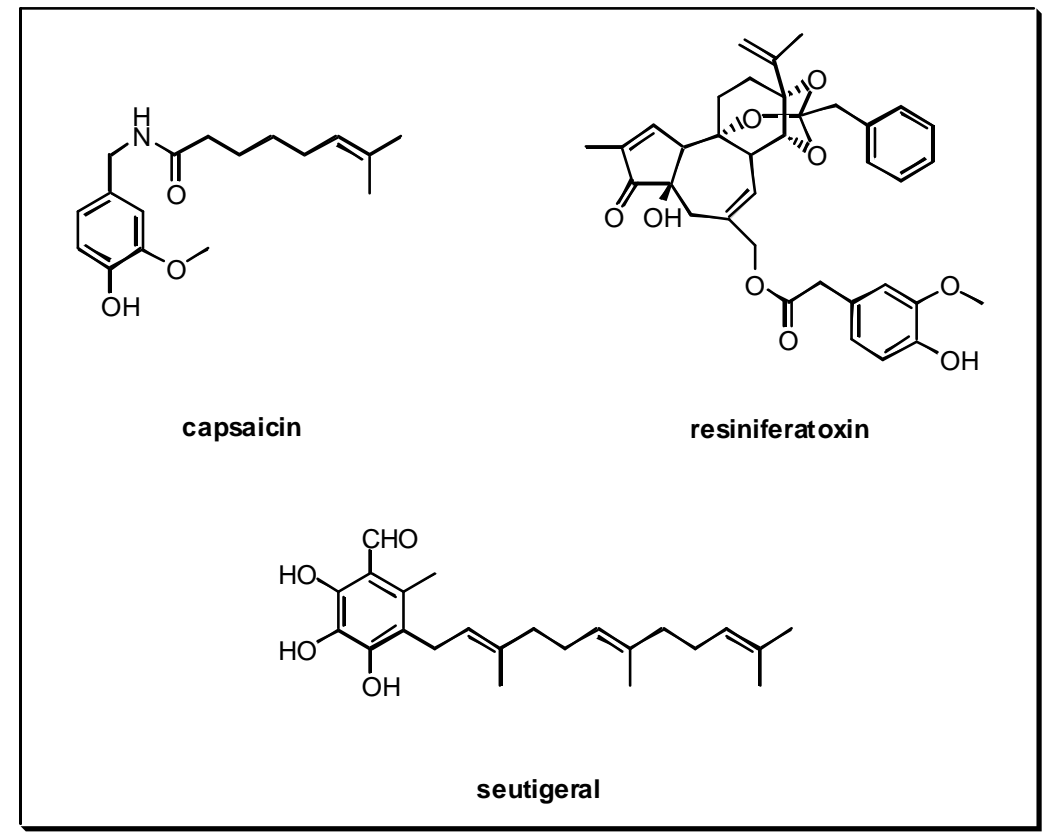

Figure 1. 


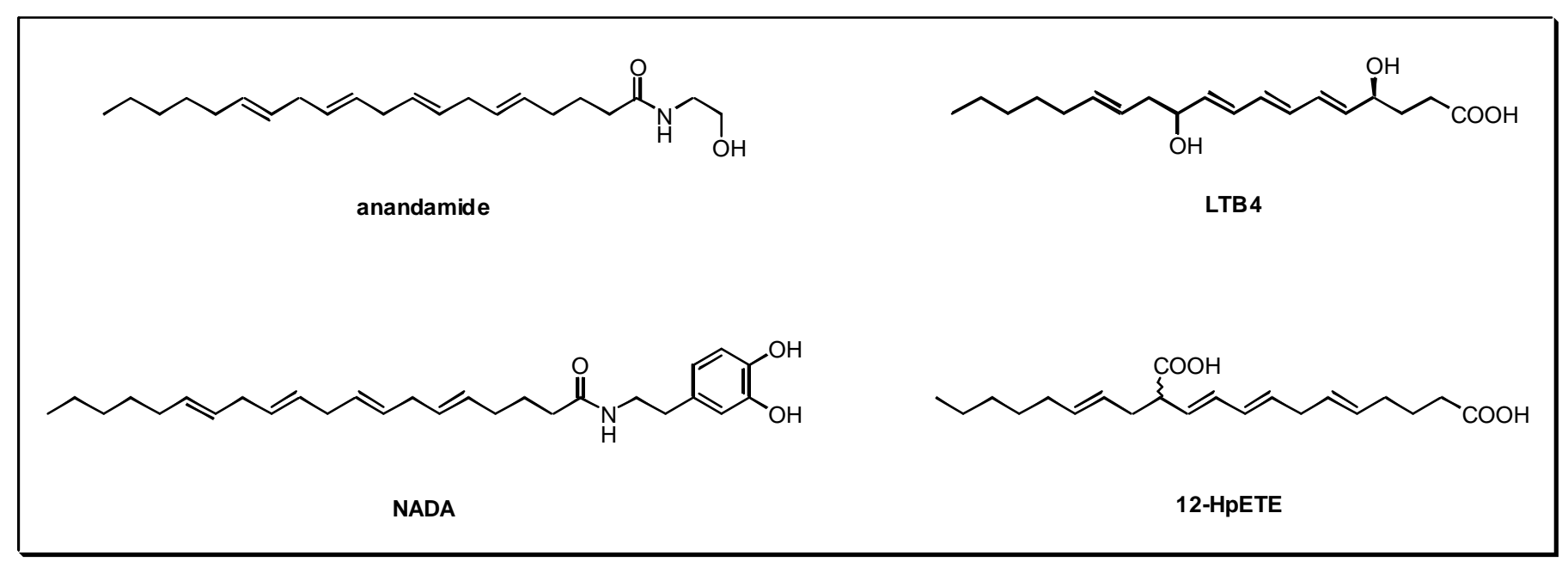

Figure 2. 


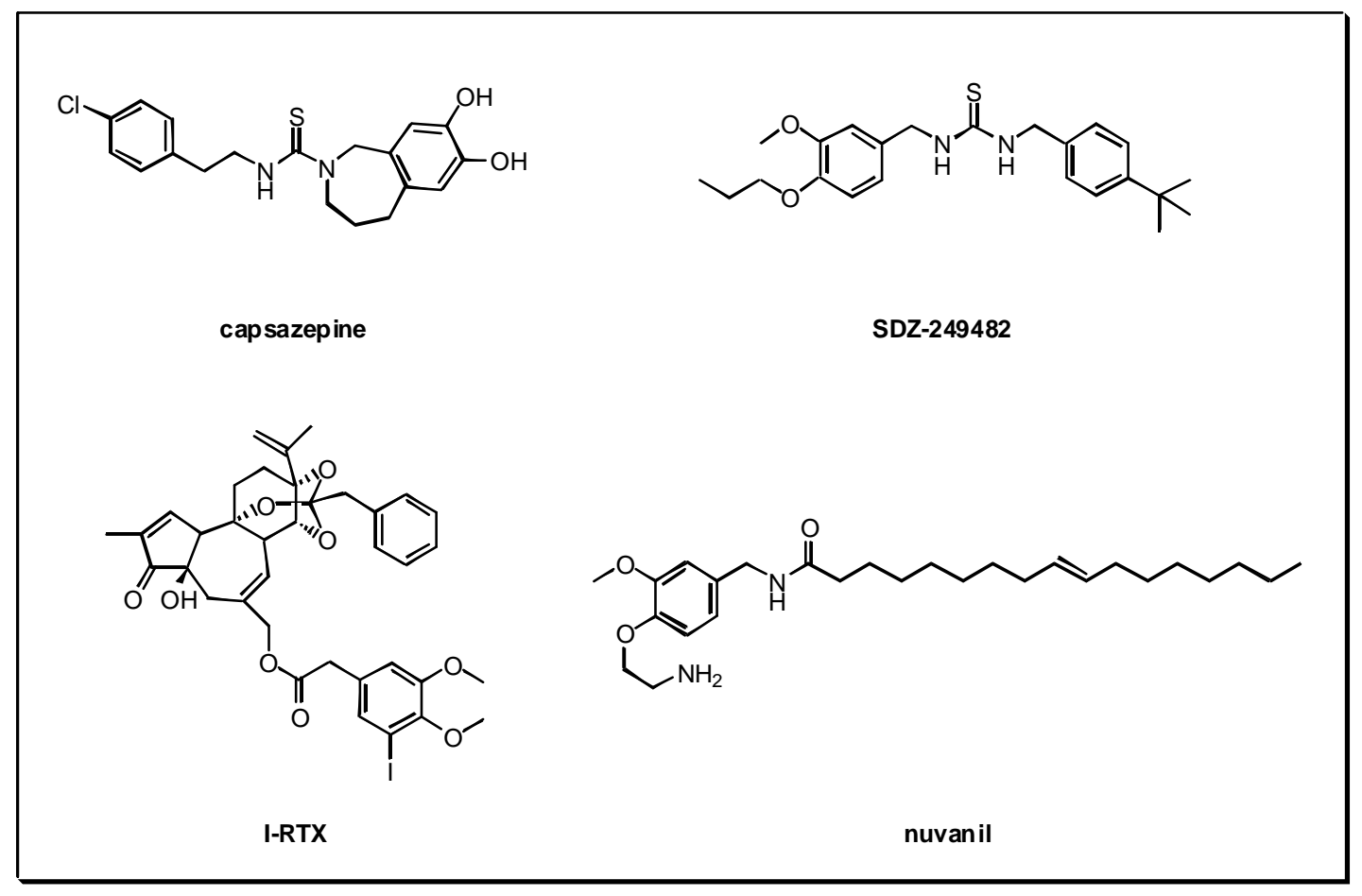

Figure 3. 


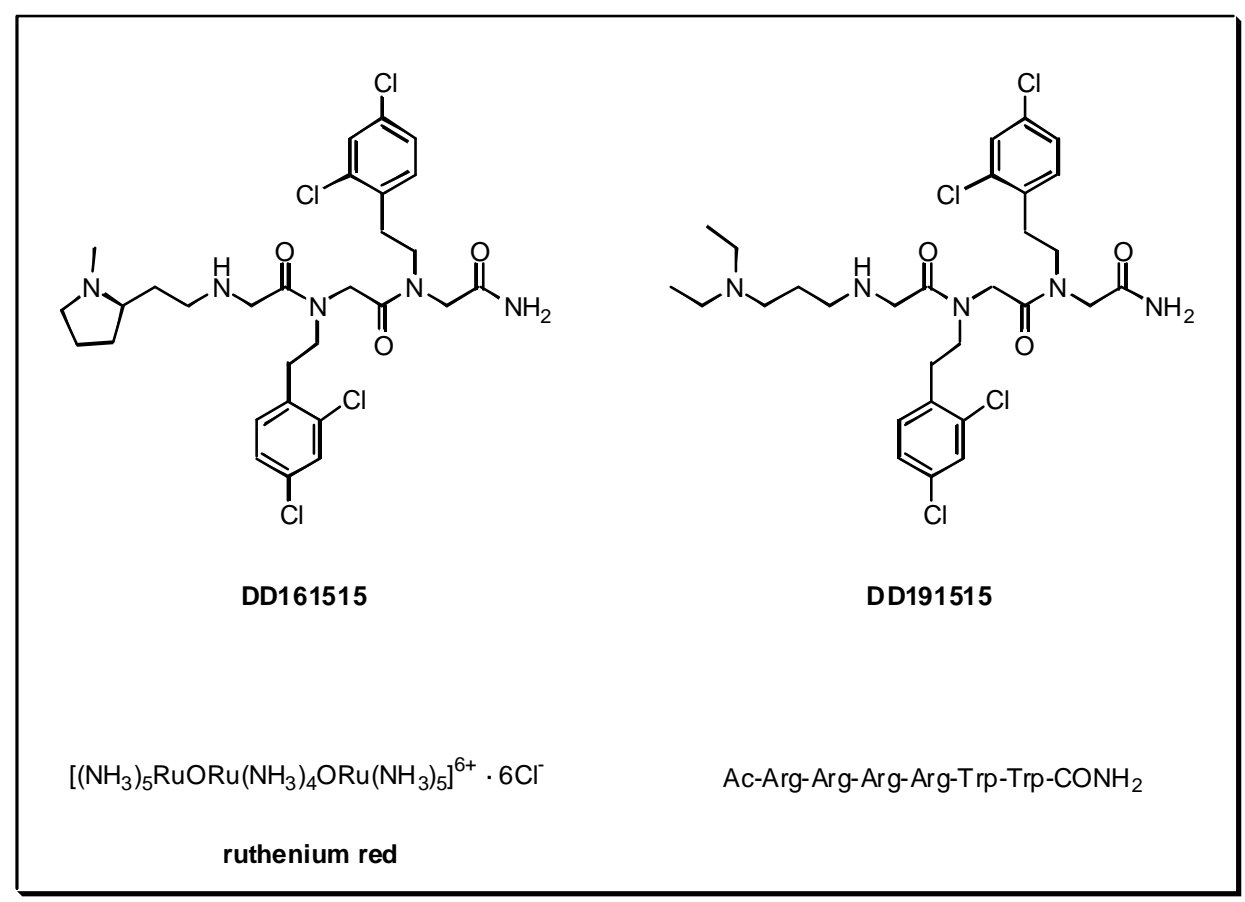

Figure 4. 


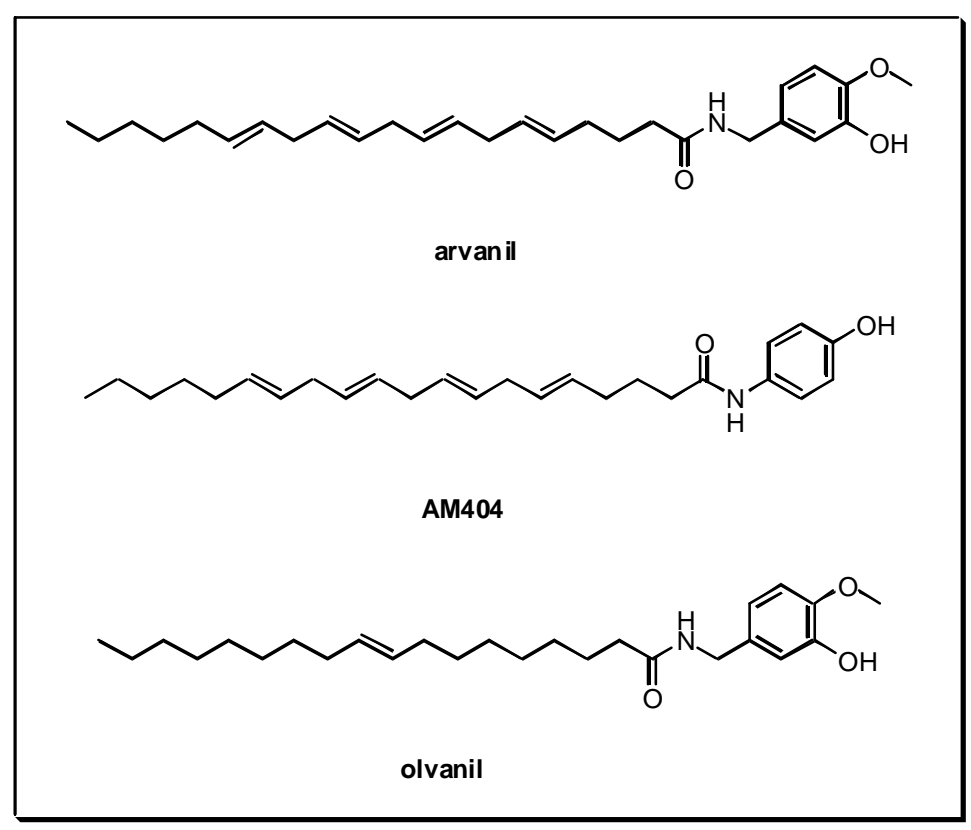

Figure 5. 